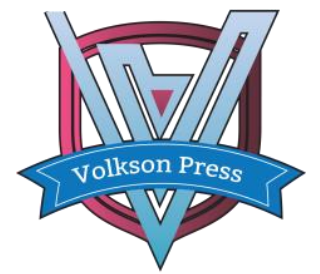

Contents List available at VOLKSON PRESS

Economics \& Management Innovations(EMI)

DOI : http://doi.org/10.26480/icemi.01.2017.373.377

\title{
Smart Planting Using Internet of Things Technology
}

\author{
Sujin Na-nakhon, Narongdech Keeratipranon and Paethai Akarajariyakul \\ * College of Innovative Technology and Engineering Dhurakij Pundit University, Bangkok,Thailand \\ *paethai.akl@dpu.ac.th
}

This is an open access article distributed under the Creative Commons Attribution License, which permits unrestricted use, distribution, and reproduction in any medium, provided the original work is properly cited.

\section{ARTICLE DETAILS}

\section{Article History:}

Received 02 october 2017

Accepted 06 october 2017

Available online 11 october 2017

Keywords:

AWS, AmazonEC2, DynamoDB, Gateway, IoT, MQTT, Sensor, Wireless

\section{ABSTRACT}

This paper examines smart farming techniques based on the Internet of Things concept to solve various problems caused by traditional agriculture such as high labor costs, improper water resource usage, and deficient control of the environment. To solve these problems, we present a model for the study of remote farming called "Smart Planting Model", which consists of sensing devices that detect humidity, temperature, water levels, and humidity in the soil. The board controller uses Arduino Uno R3, which is equipped with a set of digital and analog input/output. The remote monitoring system is available from the Amazon EC2 dashboard, which is under the service of AWS Cloud. Based on the empirical findings, we found that the developed model connected reliably to subsystems. Testing of the control system of watering, temperature and lighting can be performed automatically and accurately, as specified in the program. Control and monitoring systems can be connected and logged on from both PCs and mobile phones.

\section{Introduction}

Agriculture has been the main occupation of Thai farmers for a long time. Traditional farming is human labor. There are some limitations that make farming difficult to manage. For example, the problem of expensive labor costs is caused by the migration of people from rural to urban areas. Itis also difficult to manage water usage appropriately due to the different amounts of water needed for each plant species. Another issue is that each plant species requires different environments, such as light or temperature.

To overcome these problems, we introduce smart farming techniques to apply diverse types of sensors to observe continuously the change of environment and transfer parameters to the control system. The data are processed by a microcontroller board, where the decision is based on the conditions specified in the program that is set for each plot. With this technology, it is possible to reduce the labor costs of overseeing the plantation area as it is automated. This system can also supervise and control the factors and environment of the plots to estimate the proper use of resources. Moreover, it can control and monitor the plots from a remote distance.

\section{About the Project}

In this experiment, we have created a model for studying the remote control of agriculture, called the "Smart Planting Model", which applies the concept of the Internet of Things technology. This can communicate between smart devices and control devices, remotely. Different types of messages can also be used to exchange information with different types of sensors in order for us to manage and share information through these resources effectively using our smart planting system.

Since the world is likely to advance new technologies, it is necessary target agricultural trends. It had many projects that applied wireless sensor networks gather information from various sensors that are active at various nodes and over wireless protocols. The Information collected provides information on various environmental factors.

There are many other factors that reduce efficiency at higher levels. To solve this problem, it is necessary to develop an integrated system that will take care of factors affecting efficiency at every stage. Perfect automation in the agriculture cannot be achieved due to various problems.

The automatic remote control system consists of integrated control systems, intelligent devices, and wireless networking system. Integrated control system includes basic components, such as humidity control watering control, air blower control, and Intelligent control systems.

\section{Internet of Things (IoT)}

To access IoT technology, it is necessary to have three key components, which consist of device, gateway, and cloud, as in Figure1.

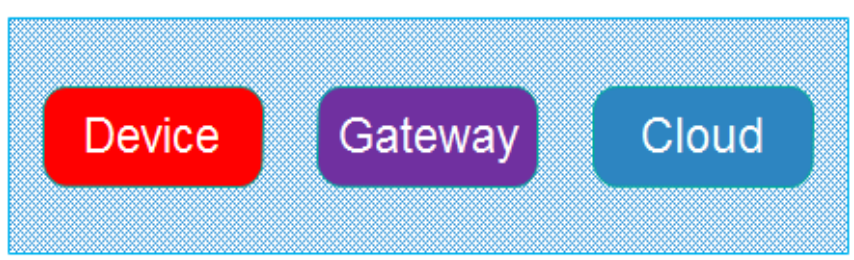

Figure. 1 IoT Components

The device consists of hardware and software that interacts directly with the world. Devices connect to a network to communicate with each other, or to centralized applications. Devices might be connected directly or indirectly to the Internet. Gateway allows devices that are not directly connected to the Internet to access cloud services. Although the term gateway has a specific function in networking, it is also used to describe a class of devices that process data on behalf of a group or cluster of devices.

\section{Smart Planting Model}

This is a service that uses networks to actualize a convergence of service in the agricultural field to cope with various problems, for example, the control of watering, temperature and lighting with the aid of information processing and autonomous control technologies of the information technology area. The overview of this model is given in Figure 2. 


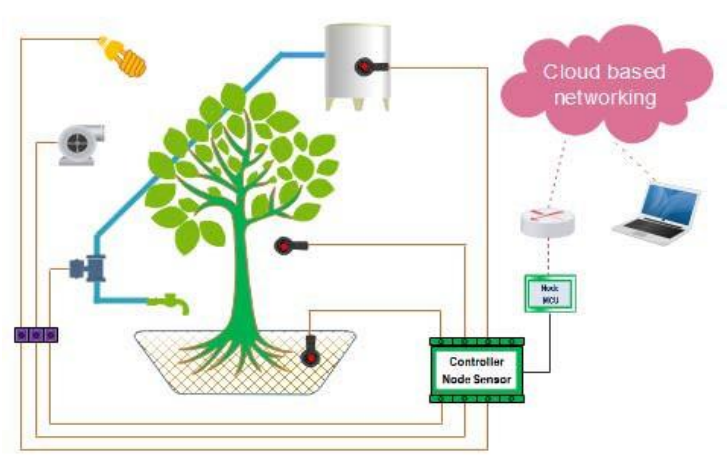

Figure. 2 Smart Planting Model

\subsection{Block diagram}

The Smart Planting Model consists of three key components (according to IoT concept) which each component are assigned, to any functional. The Arduino (Device) receives electrics for signals from any sensor after that sends data to NodeMCU, and the NodeMCU (Gateway) sends the data to AWS with MQTT protocols, with data storage in DynamoDB, and executes by EC2 ( Cloud) on a web browser with HTTP protocol, as Figure 3.

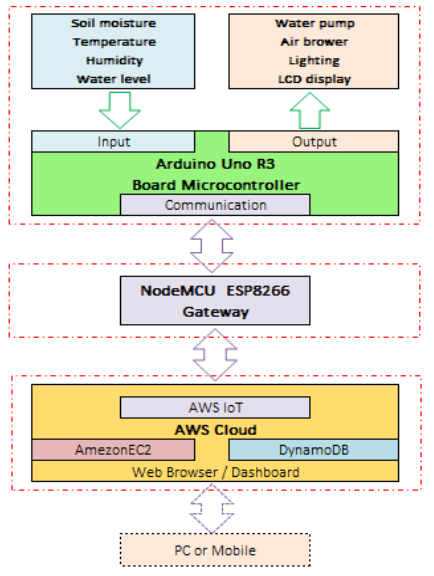

\subsection{Architecture}

Figure. 3 Block diagram

The main components used in the peper are divided into three parts:

\section{1) Devices: It is necessary to have elements:}

- Soil moisture sensor is a sensor which senses the measure of water content in soil. A soil moisture probe is made up of multiple soil moisture sensors. The digital output is fixed and the analog output threshold can be varied. It works on the principle of open and short circuits. The output status is high or low. Methods for exploiting soil dielectric properties measure proxy variables that more or less include a component due to the soil electrical conductivity, and are inherently sensitive to variations in soil salinity and temperature, as well as water. Measurements are also affected by soil bulk density and the proportion of bound and free water determined by the soil type. Nevertheless, accuracy and precision can be achieved under specific conditions, and some sensor types have become adopted widely for scientific work. When the soil is dry, the current will not pass through it, and it will act as an open circuit. Hence, the output is said to be a maximum. When the soil is wet, the current will pass from one terminal to the other, the circuit is said to be short, and the output will be zero.

- Temperature/ Humidity sensor uses a capacitive humidity sensor and a thermistor to measure the surrounding air, and spits out a digital signal on the data pin. This has been calibrated with digital signal output of the temperature and humidity combined sensor.

The specification has a power of 3 to $5 \mathrm{~V}$, and I / O can use up to $2.5 \mathrm{~mA}$ during conversion, which is suitable for reading humidity $20-80 \%$ with $5 \%$ accuracy, and for reading temperature $0-50^{\circ} \mathrm{C}$ Accuracy $\pm 2^{\circ} \mathrm{C}$ No more than $1 \mathrm{~Hz}$ Sampling rate (every second), with body size $15.5 \mathrm{~mm}$ x $12 \mathrm{~mm}$ x $5.5 \mathrm{~mm} 4$ legs and distance $0.1^{\prime \prime}$. The sensor detects signal processing within the process to call these calibration coefficients, with standard single interface.

If the temperature range is exceeded, certain range motor (blower) starts that rangs up to $30^{\circ} \mathrm{C}$. The sensors are precision temperature sensors, whose output voltage is linearly proportional to Celsius. It has an advantage over linear temperature sensors calibrated in degree Kelvin, with its output to obtain convenient Centigrade scaling.

Humidity is a measure (in percentage) of the vapor in the air compared with the total amount of vapor that could be held in the air at a given temperature of the system. The sensor is used for measuring moisture content in the atmosphere, and is based on measurements of temperature, pressure, and mass.

Water level sensor or float switch is a device used for the level of liquid within a water tank, and may actuate a pump, indicator, alarm, or other device. Float operated liquid level controls operate on the basic buoyancy principle, which states "the buoyancy force action on an object is equal to the mass of liquid displaced by the object." As a result, floats ride on the liquid surface partially submerged, and move the same distance as the liquid level moves, and are normally used for narrow level differential applications, such as high level or low level alarms.

- Sensor node, IoT is about interconnecting embedded systems, bringing together two evolving technologies, wireless connectivity and sensors. These connected embedded systems are independent microcontroller-based computers that use sensors to collect data, and are networked together, usually by wireless protocol, such as WiFi, Bluetooth, 802. 11. 4, or a custom communication system. The networking protocol is selected based on the distribution of nodes and the amount of data to be collected.

- Arduino is a microcontroller board with open-source physical computing platform based on a simple i/o board, and a development environment that implements the Processing/Wiring language. Arduino can be used to develop stand-alone interactive objects or can be connected to software on your computer (for example, Flash, Processing, MaxMSP). Open-source IDE can be downloaded for free (currently for Mac OS X, Windows, and Linux).

\section{2) Gateway}

An IoT gateway device bridges the communication gap between IoT devices, sensors, equipment, systems, and cloud. By systematically connecting the field and cloud, IoT gateway devices offer local processing and storage solutions, as well as the ability to control field devices autonomously, based on data input by sensors.

NodeMCU, the development board is a powerful solution to program microcontrollers and be part of the Internet of Things ( IoT) . The NodeMCU development board, based on ESP8266EX, is a module with a microcontroller, integrated $\mathrm{Wi}-\mathrm{Fi}$ receiver, and transmitter. NodeMCU supports several programing languages, so it is very easy to upload programs from any computer over a micro-USB port.

\section{3) AWS cloud computing}

Cloud computing is a mechanism for providing IT-related functionality as a service, allowing users to access technology-enabled services from the Internet without needing knowledge, expertise or control over the infrastructure that supports them. For example, in-the-cloud security detects and blocks threats in the cloud where they occur before they can harm computers, or networks.

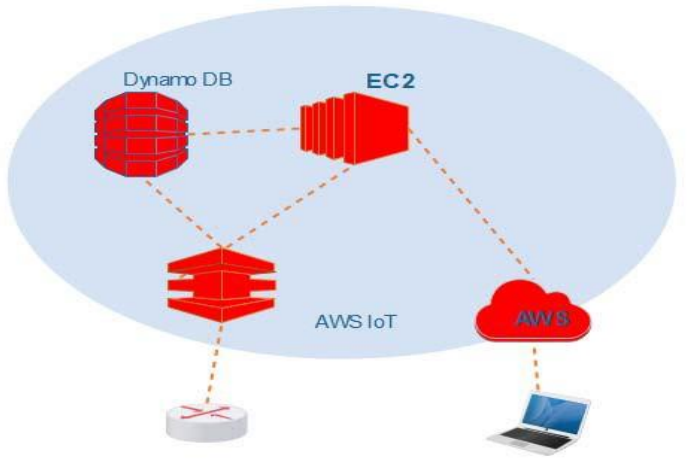

Figure. 4 AWS cloud

Figure 4 shows the Amazon Web Services ( AWS) used in this project. It is a subsidiary of Amazon. com that provides on-demand cloud 
computing platforms. These services operate from many global geographical region, including 6 in North America. They include Amazon Elastic Compute Cloud, also known as " EC2", and Amazon Simple Storage Service, also known as "S3". As of 2016, AWS has more than 70 services, spanning a wide range, including compute, storage, networking, database, analytics, application services, deployment, management, mobile, developer tools, and tools for the Internet of Things. Amazon markets AWS as a service to provide large computing capacity more quickly and cheaper than a client company building an actual physical server farm.

Amazon EC2 is an integral part of AWS, and is designed to be flexible and allow users to scale their deployed applications quickly. A user can create, launch, and terminate server-instances as needed, hence the term "elastic". EC2 provides a Query API. These requests are HTTP or HTTPS requests that use the HTTP verbs GET or POST and a Query parameter named Action. For more information about the API actions for Amazon EC2, see Actions in the Amazon EC2 API Reference.

AWS IoT is a managed cloud platform that connects devices easily and interact securely with cloud applications and other devices. AWS IoT can support billions of devices and trillions of messages, and can process and route those messages to AWS endpoints, and to other devices reliably and securely. With AWS IoT, user applications can keep track of and communicate with all devices, all the time, even when they are not connected.

DynamoDB is built to support workloads of any scale with predictable, low-latency response times. To ensure high availability and low latency responses, DynamoDB requires that the user reads and writes throughput values. It uses this information to reserve sufficient hardware resources and appropriately partitions data over multiple servers to meet the user throughput requirements. As application data and access requirements change, users can easily increase or decrease provisioned throughput using the DynamoDB console. In DynamoDB, tables, items, and attributes are the core components. A table is a collection of items, and each item is a collection of attributes. DynamoDB uses primary keys to identify each item uniquely in a table, and secondary indexes provide greater querying flexibility. The basic DynamoDB components are:

- Tables: Similar to other database systems, DynamoDB stores data in tables, or a collection of data. For example, see the table called People ( shown following) that can be uses to store personal contact information.

- Items: Each table contains multiple items. An item is a group of attributes that is uniquely identifiable among all the other items. Items in DynamoDB are similar in many ways to rows, records, or tuples in other database systems. In the example People table, each item represents a person.

- Attributes: Each item is composed of one or more attributes. An attribute is a fundamental data element, something that does not need to be broken down further. Attributes in DynamoDB are similar in many ways to fields or columns in other database systems.

\subsection{Protocols}

IoT requires a standard protocol. This project uses two types of protocols. The most promising for small devices is MQTT and HTTP, where MQTT communication between M2M and HTTP is used in webservers with users.

- MQTT was developed by Andy Stanford-Clark ( IBM) and Arlen Nipper (Eurotech; now Cirrus Link) in 1999 for the monitoring of an oil pipeline through the desert. The goals have a protocol, which is bandwidth-efficient and uses little battery power, because the devices were connected via satellite link. This was extremely expensive at that time. MQTT is the machine-to-machine protocol of the future. The minimal design makes it perfect for built-in systems, mobile phones, and other memory and bandwidth sensitive applications. It was designed as an extremely lightweight publish/subscribe messaging transport, and is useful for connections with remote locations, where a small code footprint is required and/or network bandwidth is at a premium.

For example, it has been used in sensors communicating to a broker via satellite link, over occasional dial-up connections with healthcare providers, and in a range of home automation and small device scenarios. It is ideal for mobile applications because of its small size, low power usage, minimized data packets, and efficient distribution of information to one or many receivers.

MQTT denotes MQ Telemetry Transport. It is a publish/ subscribe, extremely simple and lightweight messaging protocol, designed for constrained devices and low-bandwidth, high-latency or unreliable networks. The design principles are to minimize network bandwidth and device

resource requirements, while attempting to ensure reliability and some degree of assurance of delivery. These principles also make the protocol ideal in the emerging "machine-to-machine" ( M2M) or "Internet of Things" world of connected devices, and for mobile applications where bandwidth and battery power are at a premium.

- HTTP is an application-level protocol for distributed, collaborative, hypermedia information systems, which is the foundation for data communication for the World Wide Web (that is, internet) since 1990 HTTP is a generic and stateless protocol which can be used for other purposes, as well using extensions of its request methods, error codes, and headers. Basically, HTTP is a TCP/ IP based communication protocol that is used to deliver data ( HTML files, image files, and query results) on the World Wide Web. The default port is TCP 80, but other ports can also be used as well. It provides a standardized way for computers to communicate with each other. HTTP specification specifies how clients' request data will be constructed and sent to the server, and how servers respond to these requests.

\subsection{Systems}

The Smart Planting Model required four collaborative subsystems, namely data transmission, database storage, mechanical, and Monitoring/ Controlling, which are automatically operated and displayed. The details are described, as follows:

\section{1) Data transmission subsystem}

NodeMCU receives parameters detected by any sensor from the sensor node, and then passes through the MQTT protocol that connects to NodeMCU and AWS IoT, and transfers the data to the storage system, as shown in Figure 5.

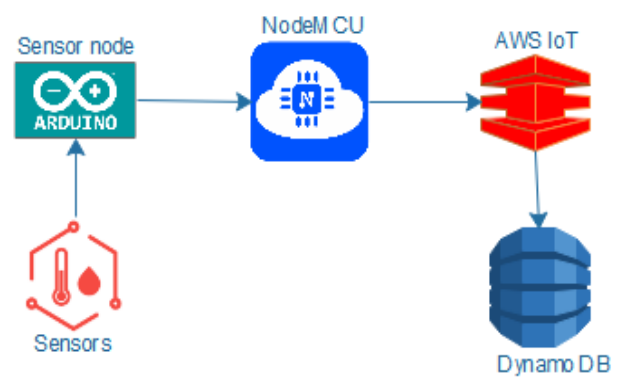

Figure. 5 Data Transmission System

\section{2) Database storage subsystem}

Data sent from the data transmission system to the database according to database tables that created under the format of DynamoDB will store in the format of JSON.

\section{3) Mechanical (output switching) subsystem}

This subsystem consists of three independentfunctions:

- Watering system, the soil humidity is measured by a moisture sensor. Measured data is sent to the control board for processing conditions or constraints. When the moisture content in the soil decreases according to the criteria set, the controller will force the solenoid valve to "On" status and release water to the plant. Similarily, when the moisture content reaches the specified threshold, the controller will switch "OFF" the solenoid valve, and the water supply is stopped.

- Temperature control system in automatic control switching "ON" and "OFF" of the temperature control system. The temperature and humidity sensor values are continuously monitored and readings are 
sent to the controller. If the temperature and humidity values exceed the threshold values, the controller will switch "ON" and the air blower will operate. Likewise, if the controller finds that the temperature and humidity are back in the set threshold, the controller will force the air blower to switch "OFF".

- Lighting control is achieved by setting the operation time through the relay to turn switch "ON" or "OFF" the lighting automatically. This system can also control the switch "ON" or switch "OF" via manual operation.

\section{4) Monitoring/Controlling subsystem}

Another feature of IoT is that the user controls and manages activity from a wireless remote distance, as well as on PC or mobile

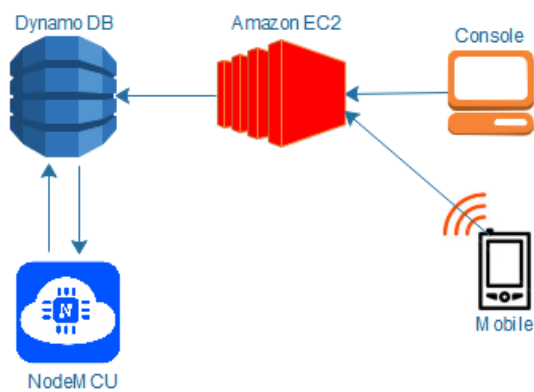

Figure. 6 Monitoring/ControllingSystem

Figure 6 shows a flow of the monitoring or control. Users can access the system via web browser of AWS with EC2. In the case of the monitoring function, EC2 will find the data stored in DynamoDB for display at the user dashboard. In addition, if the user switches to the control function, EC2 will send the request to AWS IoT and pass through into NodeMCU and controller board to enable the subsystem. Both functions communicate over HTTP protocol.

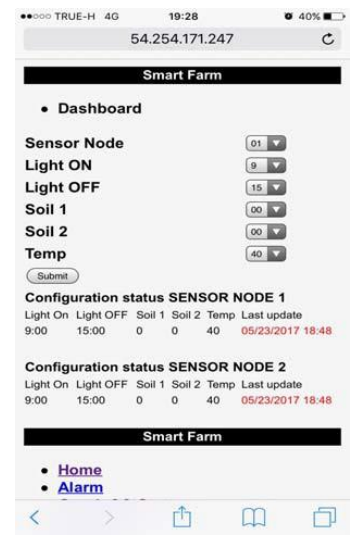

Dashboard is a user interface that resemble an automobile's dashboard, and organizes and presents information in a way that is easy to read. Figure 7 shows the information dashboard that can be monitored or controled for Humidity, Lighting, Temperature, and Soil moisture.

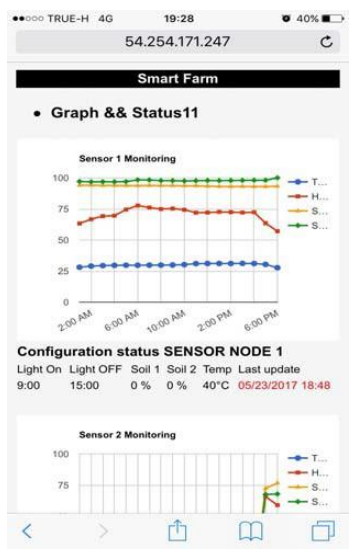

\section{Figure. 8 Graphanalysis}

Figure 8 shows the information that can be analysed as the status of each sensor node. The analysis monitors the values of the sensor node, the graph will display real time status for the values of Temperature, Humidity, Soil moisture No1, and Soil moisture No2. The access to graph monitoring can be via a fixed computer or mobile phone.

\section{Results}

This experiment has four complementary subsystems. The results of this analysis will be classified into the following sections:

- Data transmission system can connect communication links between the components.

- Mechanical system: watering systems can be activated when the soil moisture content is less than $30 \%$, and when the humidity is increased to $30 \%$, the system stops the process. Temperature control, the fan operated when the ambient air temperature and humidity in the vicinity of the monitored plot, is above 28 degrees Celsius, and stops working, when the temperature drops to 28 degrees Celsius. The water level system can be sent to an alarm in the dashboard.

- Database storage system stores the database according to the format of the DynamoDB service.

- Monitoring/ Controlling system: the dashboard displays values of all subsystems, and the command can be successfully sent to the board controller. Both PC and mobile can be applied.

\section{Discussion}

Refeing to the information obtained from the results, we discussed in each subsystem, where it was found that the overall system worked, as described in the manual. However, some of them could be improvement, as discussed below.

- Position and number of sensors installed may affect the accuracy of the data. Proper positioning and numbering should ensure that the data are accurate to meet the requirements.

- Since the process of supplying water to the plant uses a method of closing or opening valves, it can result in an inability to control the amount of water flows. It may be necessary to have a more controllable process to determine the amount of water dispensed.

\section{Conclusion}

This paper presents an IoT based design of smart planting system. The model operates for data gathering and transmission using wireless internet under the Amazon Web service. Preliminary tests show that the model is capable of monitoring and controling devices in the deployed environment, and has several advantages in term of fast delivery, zero data lose, low cost, flexibility, and user friendliness. In terms of user interfaces, they are simple and intuitive. The project also help farmers in real time monitoring of crops and effective usage of water for watering by identifying soil moisture, and is able to control the ambient temperature by identifying temperature and humidity. In addition, this model helps to monitor or control plants from remote places.

\section{Future Scope}

The future scope of the paper is to enhance the system with several required applications. One such application is to detect soil parameter, and suggests proper fertilization and feed time. Such sensors can be incorporated into the design. It can also be designed to detect particular diseases on plants and suggest proper curative measures. It is possible to predict weather if the system is made to communicate with the nearest weather station through satellite communication. These systems will be implemented in continuous demonstrations of farming and agriculture. Data services are being improved to support other type of products and provide additional services. Utilizing IoT technology, the efficiency of crops has been improved significantly, with continuous improvements that can lead to agricultural or plant production into a new age.

\section{References}

[1] Awati J. S. , Patil V. S. , " Automatic Irrigation Control by using wireless sensor networks", Journal of Exclusive Management Science - June 2012-Vol 1 Issue 6.

[2] D. D. Chaudhary, L. M. Waghmare., “ Application of wireless sensor 
networks for greenhouse parameter control in precision agriculture", International Journal of Wireless \& Mobile Networks Vol.3, February 2011.

[3] Kelly, S. D. T. , Suryadevara, N. K. ; Mukhopadhyay, S. C. " The Implementation of IoT for Environmental Condition Monitoring in Homes", Sensors Journal, IEEE, Volume:13, pp- 3846

- 3853, May 2013.
[4] White Paper: " Internet of Things Strategic Research Roadmap",Antoine de Saint-Exupery, 15 sep 2009.

[5] Zhang Feng Yulin University Yulin University tfnew21@sina.com, “ Research on water-saving irrigation automatic control system based on Internet of things Institute of Information Technology", 2011 IEEE 